\title{
Intervención de kinesiología en individuos con sobrepeso u obesidad: análisis de la composición del cuerpo y calidad de vida
}

\author{
Fabiano Leichsenring-Silva ${ }^{1 *}$; Viana Abraão-Andrade Silva ${ }^{1 ;}$ Juliana Tavares-Maciel ${ }^{1}$ \\ ${ }^{1}$ Faculdade Adventista da Bahia, Northeast Brazil CollegeBR-101, km 197, Capoeiruçu \\ Caixa Postal 18, Cachoeira Bahia, CEP: 44.300-000 - Brazil
}

\begin{abstract}
RESUMEN
Como una de las principales causas de muertes, la obesidad es una condición de exceso de grasa que acompaña a una constelación de comorbilidades que, al mismo tiempo, aumentan el riesgo de enfermedad cardíaca o cáncer. La evaluación de la composición corporal ha logrado probada mejoría con la práctica regular de actividad física. Se trató de responder en qué forma un programa de intervención primaria puede influir en la composición corporal y la calidad de vida de las personas con sobrepeso u obesidad, con el objetivo principal de analizar la composición corporal de las personas con sobrepeso. Además adaptar el uso de ejercicio, junto con ocho agentes naturales. La muestra fue compuesta por individuos de edades comprendidas entre los 18 y 30 años, sometidos a evaluación antropométrica, funcional y de calidad de vida. El protocolo de intervención fue realizado en ocho semanas, resultando en la reducción del porcentaje de grasa, reducción del peso corporal y reducción de índice de masa corporal.
\end{abstract}

Palabras clave: Obesidad, composición corporal, calidad de vida, ejercicio.

\begin{abstract}
As one of the leading causes of preventable death, obesity is a condition of excessive fat that accompanies a constellation of other morbidities that in parallel increases risk of heart disease and/or cancer. The body composition assessment has achieved proven improvement with regular practice of physical activity. This research seeks to answer how a program of primary intervention can influence body composition and quality of life on overweighted/obese individuals, with the main objective to analyze body composition in overweight individuals. This work seeks to tailor the use of physical exercise together with seven natural agents. The sample consisted of individuals aged between 18 and 30 years, who underwent anthropometric, functional and quality of life evaluations. The intervention protocol was conducted during eight weeks, resulting in reduction of body fat percentage, body weight reduction, reduced BMI and improved quality of life.
\end{abstract}

Keywords: Obesity, corporal composition, quality of life, exercise

\footnotetext{
*Correspondencia: leichs@gmail.com,(75) 3425-8096
} 


\section{INTRODUCCIÓN}

La obesidad se ha convertido en un problema de salud pública y está fuertemente asociada con muchas enfermedades crónicas no transmisibles, entre ellas: hiperlipidemia, hipertensión, resistencia a la insulina, dislipidemias, diabetes mellitus, entre otros. La mayoría de estas enfermedades se relacionan con mayor adiposidad visceral, y las principales causas de esta acumulación excesiva son la ingesta de grasa y estilo de vida sedentario. Para minimizar estos efectos nocivos, la práctica de actividad física se ha convertido en una parte importante en las estrategias no farmacológicas para controlar la obesidad. (ZAMBON et al., 2009). Así, la propuesta de este estudio fue analizar la composición corporal de personas con sobrepeso u obesos que reciben kinesioterapia, a través de un programa que apunta a mejorar la calidad de vida.

Por lo tanto, fueron hechas tres tipos de evaluaciones, como estas: evaluación de la composición corporal, evaluación kinésica con énfasis cardiovascular y análisis de calidad de vida a través del cuestionario WHOQOL-bref, siendo todas ellas llevadas a cabo antes y después del programa de intervención.

Estudios realizados por la Asociación Brasileña para el Estudio de la Obesidad y el Síndrome Metabólico (2012) muestra que el costo total para SUS, estimado por un año con todas las enfermedades relacionadas con el sobrepeso y la obesidad como el cáncer, diabetes, enfermedades del corazón, entre otras, es de U\$ 20,2 billones. La hospitalización cuesta aproximadamente U\$ 1,5 billones y los procedimientos ambulatorios U\$\$ 680 millones. En las últimas décadas, la obesidad ha avanzado hasta convertirse en una epidemia mundial. La Organización Mundial de la Salud (OMS) estima que, en el año 2005, fueron aproximadamente 1,6 billones de adultos con sobrepeso y, al menos, 400 millones eran obesos. Asimismo predijo que, en el año 2015, aproximadamente 2,3 billones de adultos tendrán sobrepeso y más de 700 millones serán obesos.

Wilmore y Costill(2001) informan que el sobrepeso es el peso que supera el peso normal para su altura y su constitución física, ya que la obesidad se refiere a la condición donde el individuo tiene una cantidad excesiva de grasa corporal. Ya Oliveira (2003) dice que la obesidad es un exceso de grasa corporal a la masa corporal magra, y el sobrepeso es una proporción relativa de peso mayor que la deseable para la altura. Hay mucha confusión sobre el significado preciso de los términos exceso de peso (overweight), exceso de grasa (overfat) y obesidad, cuando aplicada a la composición corporal y relacionada con riesgos de la salud respecto a una grasa corporal excesiva. McArdle (2008) habla que cada término tiene un significado diferente, dependiendo de la situación y el contexto de su uso. En la mayoría de la literatura médica, el término overweight describe una condición de exceso de grasa, incluso en la ausencia de medidas concomitantes de la grasa corporal.

Según Lee (2007), la obesidad humana es un aumento en el número de TNF- $\alpha$ en el tejido adiposo, los sujetos obesos tienen una expresión dos veces más grande para estos receptores de la célula que los no obesos. El TNF- $\alpha$ desempeña un papel importante en la regulación del metabolismo del tejido adiposo, así como en su distribución. El gen del TNF- $\alpha$ parece influir en la distribución de la grasa corporal según el sexo, en los hombres el efecto más significativo se expresa en la circunferencia abdominal y en el espesor del pliegue de piel suprapúbica. En las mujeres el impacto está en la raíz de la circunferencia del muslo y en el pliegue cutáneo de esa región. El aumento de peso para llegar al sobrepeso y a la obesidad también se los ha relacionado a enfermedades del corazón y la calidad de vida.

Investigando a adultos con coronariopatía, Milani et al. (2004) observaron significativos cambios en el estilo de vida, la incidencia de factores de riesgo y los niveles de PCR después de 12 semanas de rehabilitación cardiovascular. Sin embargo, Barbeau et al. (2002) en un estudio controlado con 74 adolescentes obesos sometidos a un programa de ocho meses con ejercicio aeróbico y asesoramiento nutricional, mostró débil asociación entre adiposidad, condición física, marcadores inflamatorios de fibrinógenos y PCR.

Aunque existan pocas dudas en cuanto a la mejora en la calidad de vida y, sobre todo, en el estado de salud mediante un programa de ejercicio físico en obesos, esos beneficios dependen de una prescripción del ejercicio adecuado en cuanto a su intensidad, duración, frecuencia y modo. (KISNER, 2005). Las actividades aeróbicas generan efectos directos y indirectos sobre el sistema circulatorio, la calidad de vida, tolerancia al estrés, hábitos de vida, la flexibilidad y la fuerza del músculo. (ROSSETTI, 2009). Wilmore y Costill (2009) encontraron que la práctica de ejercicio físico esta asociada con una oferta satisfactoria de energía, permitiendo un mayor uso de proteína en la dieta y disminución de grasa corporal. Varias otras influencias positivas están relacionadas con la actividad física regular, entre estas el aumento en el desarrollo musculo-esquelético, masa corporal magra, mejora de los niveles de resistencia cardiorrespiratoria, eficiencia muscular, además a 
los efectos psicosociales importantes. A pesar de que los ejercicios por sí solos no pueden ser capaces de promover la rápida pérdida de grasa, proporcionan muchas ventajas sobre otros tipos de tratamiento, como el mantenimiento de un mejor estilo de vida.

Estudios epidemiológicos y clínicos realizados, por Ciolac y Guimarães (2004), han demostrado que la práctica regular de actividad física es un factor importante para la prevención y tratamiento del síndrome metabólico -también conocido como síndrome $\mathrm{X}$, síndrome de resistencia a la insulina, síndrome plurimetabólica o cuarteto mortal- que se caracteriza por la agrupación de factores de riesgo cardiovasculares como hipertensión, resistencia a la insulina, hiperinsulinemia, intolerancia a la glucosa, diabetes tipo 2, obesidad central y dislipidemia (colesterol LDL alto, triglicéridos altos y colesterol HDL bajo).

Es esencial que los funcionarios de salud pública promuevan la calidad de vida, de modo que podemos reducir la vulnerabilidad y riesgos para la salud relacionados con sus factores y determinantes como modos de vida, condiciones de trabajo, vivienda, medio ambiente, educación, ocio, cultura, acceso a bienes y servicios esenciales. Para la OMS, calidad de vida es la percepción individual de la posición en la vida, en el contexto de su sistema de cultura y valor, en la que se inserta y en relación con sus objetivos, expectativas, normas y preocupaciones.

Hay, por lo tanto, la necesidad de cultivar hábitos saludables como comer correctamente, mantener el equilibrio emocional en la familia, en el entorno social y profesional y, sobre todo, estar activo. Para obtener resultados satisfactorios debe existir el paralelismo entre el programa de ejercicios y la dieta. Un programa solo llega a ser eficaz si es capaz de cambiar hábitos de vida e incorporar, en definitiva, una propuesta para una vida más saludable. (BARROS, 2002). Ahí está la importancia del programa propuesto en este estudio, donde se evaluaron la composición corporal y la CDV antes y después de ocho semanas del cambio en el estilo de vida, como un factor que contribuye en la mejora de la composición corporal y la calidad de vida de las personas con obesidad/sobrepeso.

\section{MATERIAL Y MÉTODOS}

Se trata de una estudio experimental o intervencionista (Marconi y Lakatos, 2001), que tuvo lugar en la comunidad académica de la Universidad Adventista de Bahia (FADBA) en individuos con sobrepeso $u$ obesidad. La investigación fue aprobada por el comité de ética de la investigación de FADBA, siguiendo las recomendaciones de la resolución 196/96 del Consejo Nacional de Salud, bajo el CAAE 0036.0.070.000.10.

La muestra estuvo constituida por 17 personas que tenían un Índice de Masa Corporal (IMC) entre 25 y 29,9 , con sobrepeso o entre 30 a 34.9 y con grado I de obesidad (McArdle, Katch y Katch, 2008), escogidas por conveniencia que tenían IMC con sobrepeso $\mathrm{u}$ obesidad de grado 1, con edades entre 18 y 30 años. Los criterios adoptados para la exclusión del programa fueron: (a) la negativa a participar en la recolección de datos, (b) cualquier problema físico o mental que temporal o permanentemente le impidan de realizar las actividades relacionadas con el programa, (c) personas que tienen enfermedades del corazón, (d) personas que no han firmado el consentimiento informado. La muestra fue evaluada antes de iniciar el programa y al final del mismo.

Los participantes fueron referidos a la evaluación antropométrica y funcional antes de la realización del protocolo de actividad física. Se utilizó el protocolo para la medición del porcentaje de grasa el peso, altura, IMC, pliegues cutáneas subscapular, axilares media, tríceps braquial, muslo, pecho, abdominal y suprailíaco; los diámetros de hueso bicondilar del húmero, bicondilar del fémur y biestiloideo; el porcentaje de grasa, porcentaje del peso del hueso, porcentaje de masa corporal magra, porcentaje del peso muscular, porcentaje de peso residual, peso de la grasa, peso de masa corporal magra, peso del hueso, peso muscular y peso residual. Como herramienta para la verificación de la CDV fue utilizado el cuestionario de la Organización Mundial de la Salud (Ginebra, 1995) validado para la población brasileña WHOQOL-bref, que representa a los dominios físicos, psíquicos, social y medioambientales.

El protocolo de intervención fue realizado en ocho semanas, donde cada participante tenía de manera personalizada la prescripción del ejercicio. El tipo de ejercicio realizado era el aeróbico, con duración de 20 a 40 minutos, respetando la intensidad de la propuesta, que van desde leve a moderado. La tasa fue de tres a cinco veces a la semana, y el modo fue elegido por el participante, ejercicios que le gustaban realizar. Hemos propuesto a los participantes un cambio en el estilo de vida para los agentes naturales, efectivos en la promoción y preservación de la salud, siendo el uso adecuado de agua, aire puro, ejercicio físico, temperancia, descanso suficiente, luz del sol, nutrición adecuada y espiritualidad. Mediante la incorporación de estos agentes profilácticos en los hábitos de vida pretendemos iniciar una conservación eficaz del plan de salud por medios naturales, puesto 
que además de prevenir la enfermedad, estos agentes naturales potencialmente contribuyen a la curación.

Para el análisis de datos, fueron calculados los promedios y desviaciones estándar de los promedios para cada una de las medidas realizadas. Asimismo, se utilizó el programa SPSS versión 17.0 para Windows. Las diferencias se consideraron significativas cuando el análisis estadística presentaba $<\mathrm{p} 0.05$.

\section{RESULTADOS Y DISCUSIÓN}

La obesidad ha alcanzado proporciones epidémicas $\mathrm{y}$, desde el punto de vista de la salud pública, la obesidad resulta en un gasto muy alto, principalmente por el hecho de estar relacionada a un gran número de enfermedades y factores de riesgo para las enfermedades cardiovasculares. Los resultados revelan una disminución en el peso y en el IMC después de un programa de intervención primaria de ocho semanas, asociadas con agentes profilácticos de buena CDV. Además de las variables mencionadas también podemos citar el pliegue cutáneo subscapular que tuvo una mayor reducción, en comparación con otros seis pliegues evaluados en el programa.

Entre las 17 personas que iniciaron el estudio, solo seis han completado el proceso de intervención de ocho semanas, y fue realizada la evaluación final de todos los parámetros investigados. Todas las personas que no participaron en la encuesta alegaron razones personales como estudio o trabajo, entre otros, no existiendo pérdidas por intervención del protocolo de ejercicios en la salud de estos. La muestra inicial estaba compuesta por $64 \%$ de las mujeres (11) y $36 \%$ de hombres (6) y en la muestra final tenía 50\% hombres y $50 \%$ mujeres. La edad promedio fue de $2.57 \pm 21$.

Después de realizado el entrenamiento, de ocho semanas con ejercicio aeróbico de baja o mediana intensidad durante tres veces por semana, se puede observar una caída de los valores del peso (Figura 1) y del IMC (Figura 2).

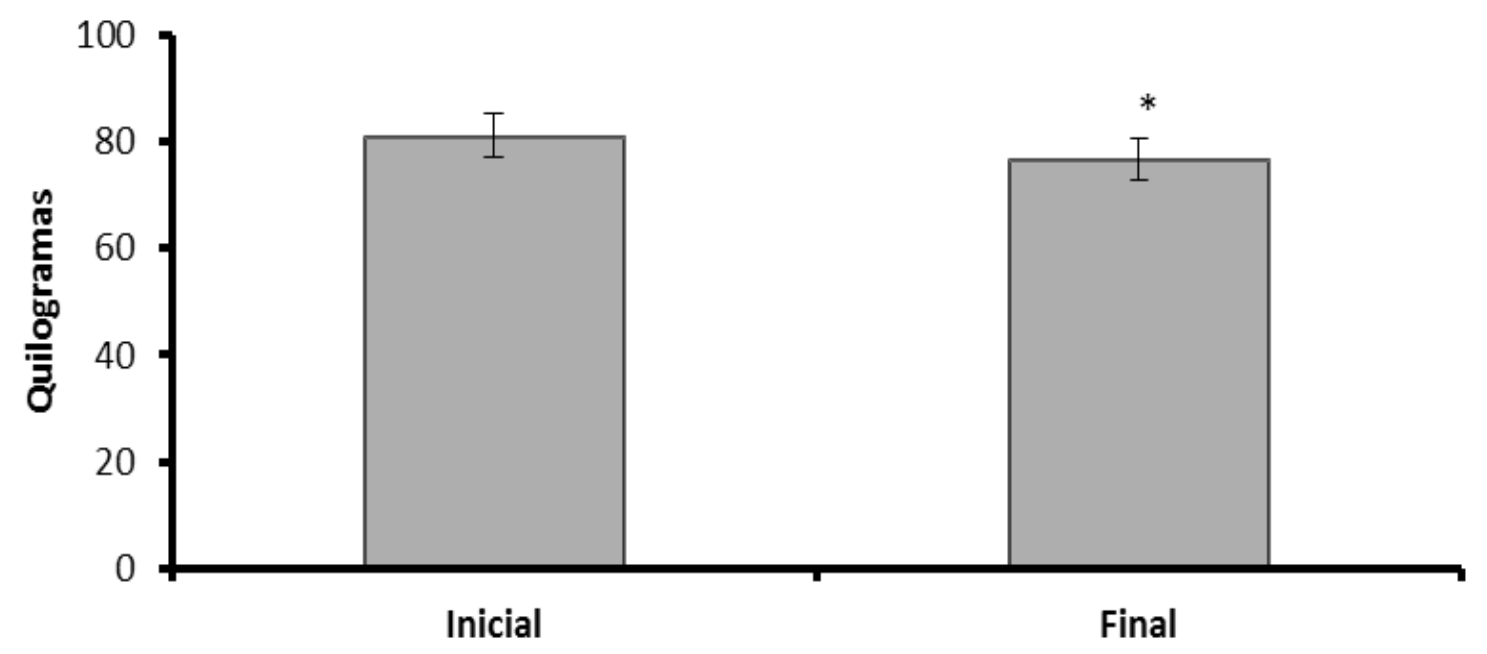

Figura 1.

Peso de la muestra antes y después del programa de entrenamiento. Datos expresados como media con barra de error estándar de la media. Prueba t pareada. $* \mathrm{p}<0.05$.

En la figura 1 vemos una reducción en la masa corporal, y obsérvese que al inicio del programa la muestra tenía un promedio de $81.05 \mathrm{Kg}$ de peso corporal total y después de la evaluación final fue de $76,5 \mathrm{Kg}$ de peso corporal total, lo que representa una pérdida de $5,62 \%$, comparado con el peso visualizado inicialmente. El IMC (Figura 2) hemos visto que hubo una reducción de 5,61\% del valor medido antes del programa, en relación con el valor medido al final del mismo. El promedio del IMC inicial era de $28,92 \mathrm{Kg} /$ $\mathrm{m}^{2}$ y el final fue $27,3 \mathrm{Kg} / \mathrm{m}^{2}$. Observamos en el final del programa una disminución de $27,71 \%$ del pliegue cutáneo subscapular (Figura 3 ) en relación con el valor inicial, dando por resultado 22,17 milímetros de grosor, con la media inicial que era 30,67 milímetros. 


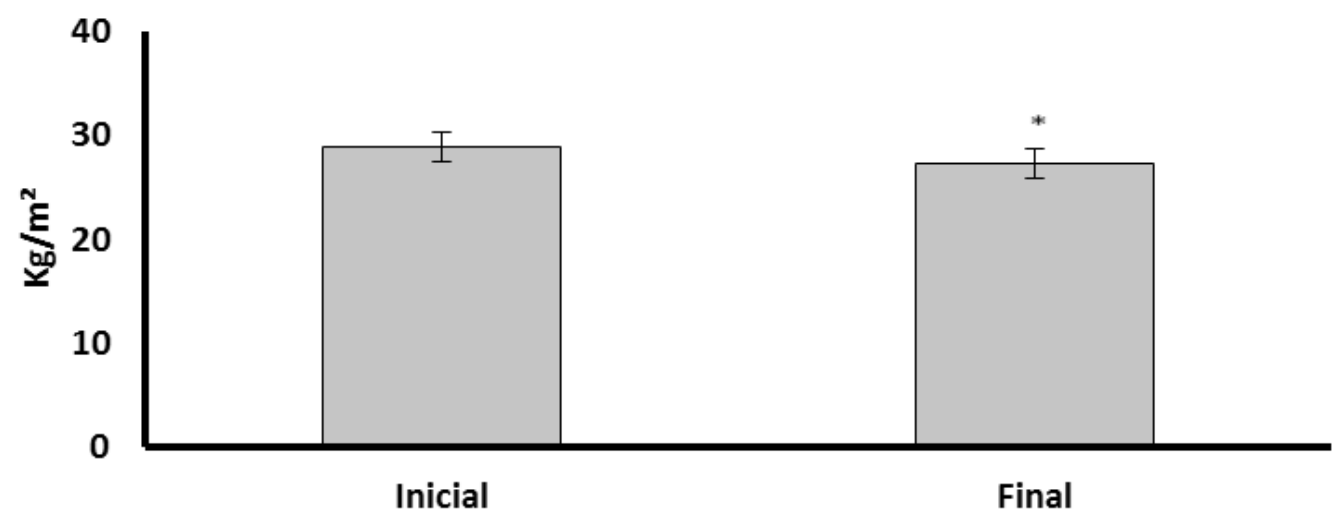

Figura 2.

Índice de masa corporal de la muestra antes y después del programa de entrenamiento. Datos expresados como media con barra de error estándar de la media. Prueba t pareada. $* \mathrm{p}<0.05$.

Según estudio de Glaner (2006) la composición del cuerpo es la técnica que se ha utilizado en todo el mundo para la obtención de índices, tales como el porcentaje de grasa corporal, IMC. Además de ser una técnica más barata, muestra una fiabilidad excelente. Esta técnica hace uso de medidas lineales, masa, diámetro, perímetro y pliegues donde pueden utilizarse solos o en combinación. La técnica de medir el porcentaje de grasa de los pliegues cutáneos se ha utilizado en el mundo, por el hecho de que tuvo gran aceptación entre los investigadores. También debido a que obtuvo el porcentaje de grasa de la técnica antropométrica asociados muy bien y no difiere significativamente el porcentaje de derivados del pesaje hidrostático, que se toma como criterio de validación de otras técnicas.

La masa corporal tuvo una reducción significativa en nuestro estudio. Tomamos nota de que el peso promedio de la muestra fue de $81.05 \mathrm{Kg}$ antes de la intervención. Al final de las ocho semanas observamos que este promedio se redujo a 76,5 $\mathrm{Kg}$. En un estudio similar, realizado con 40 mujeres divididas en dos grupos, el peso corporal tuvo una reducción durante el período de la intervención.

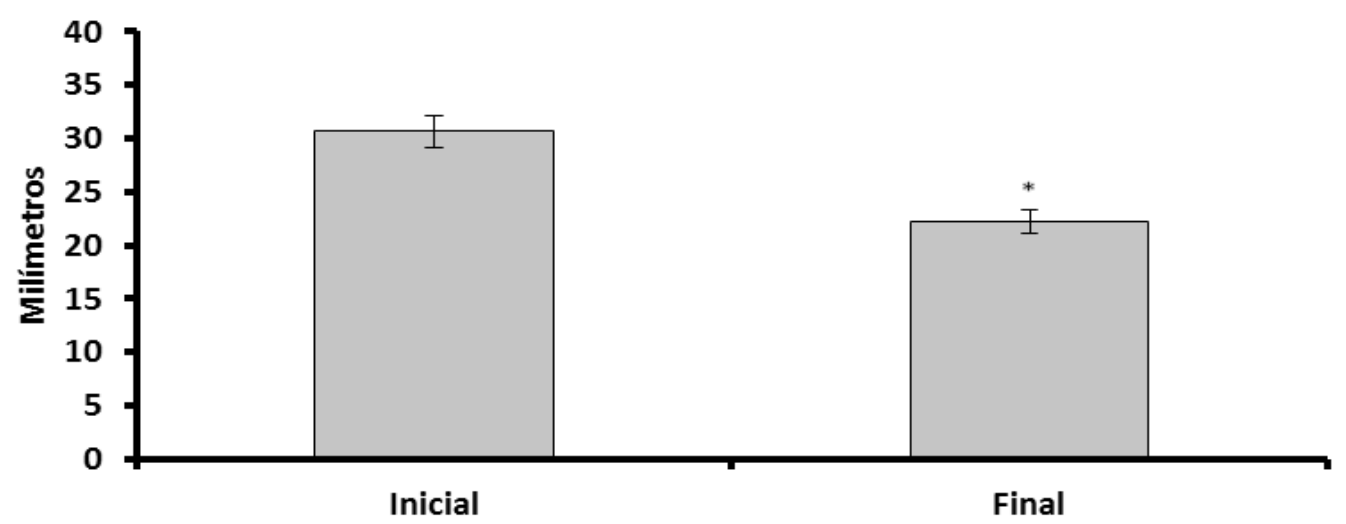

Figura 3.

Índice de masa corporal de la muestra antes y después del programa de entrenamiento. Datos expresados como media con barra de error estándar de la media. Prueba t pareada. $* \mathrm{p}<0.05$.

Mediano et al. (2007) reportaron una mayor reducción del peso corporal en individuos que realizan ejercicios físicos regulares, en comparación con quienes solo hacen dietas y reciben apoyo psicológico durante el período de 10 semanas. Según Costa et al., (2011) los hábitos de vida de la sociedad moderna, caracterizada por el alto consumo de dietas no balanceadas y ejercicios físicos bajos, han producido muchas implicaciones para la salud de la población, con un aumento en la incidencia de enfermedades crónicas no transmisibles, tales como diabetes, resistencia a la insulina, síndrome metabólico y obesidad. De esta manera, la importancia de cultivar hábitos saludables para así iniciar un programa de intervención primaria en individuos con obesidad o sobrepeso. En nuestra investigación buscamos alertar 
y capacitar a los individuos a modificar hábitos de vida. Por eso, trabajamos para crear conciencia sobre la explotación de recursos naturales como agua, aire puro, ejercicio físico, la temperancia, el descanso suficiente, la luz solar, la nutrición adecuada y la espiritualidad. En un estudio similar al nuestro Rocca et al (2008), estudiando los factores de riesgo en las mujeres obesas, no encontró cambios en la composición de la muestra después de 12 semanas de entrenamiento, citando que posiblemente ese intervalo de tiempo no es suficiente para que puedan observarse cambios relacionados con composición corporal. Lo que difiere de nuestro estudio que en un tiempo de ocho semanas sí estadísticamente se observaron cambios significativos no solo en las mujeres sino también en hombres con sobrepeso $\mathrm{u}$ obesidad. En nuestro estudio comprobamos que podemos conseguir un mejor control del sobrepeso u obesidad, cuando se añade los ocho agentes de la naturaleza.

La pérdida de peso tiene un gran impacto en el síndrome metabólico (FORD, 2005), Zanette (2010), el síndrome metabólico es definido como un conjunto de anormalidades metabólicas, especialmente sobrepeso, obesidad, resistencia a la insulina, dislipidemia y la hipertensión. Datos publicados revelan que la reducción del $7 \%$ al $10 \%$ del peso inicial es suficiente para reducir la circunferencia de la cintura, perfil lipídico y azúcar en la sangre. La obesidad y síndrome metabólico son consideradas enfermedades relacionadas a la inflamación crónica, y esa inflamación es reconocida como un componente de la aterosclerosis y, recientemente, algunos componentes del síndrome metabólico se han divulgado como marcadores inflamatorios. Este proceso inflamatorio puede identificarse por altos niveles de IL- 6 y TNF- $\alpha$. El TNF- $\alpha$ y la IL-6 actuando como mediadores presumiblemente como inflamatorios en trastornos metabólicos. La PCR es un marcador del proceso inflamatorio en personas. Los niveles séricos de PCR de alta sensibilidad tienen relación directa con la intensidad de la obesidad. Ford et al. (2005) estudiaron la asociación entre el síndrome metabólico y PCR y observaron que el síndrome metabólico se asoció con altas concentraciones de PCR. Estas citoquinas, en su mayoría, están relacionadas, directa o indirectamente a procesos que contribuyen a la ateroesclerosis, hipertensión, resistencia a la insulina y diabetes mellitus tipo 2 y dislipidemias, es decir, pueden representar el vínculo entre la obesidad, síndrome metabólico y enfermedad cardiovascular.

De los resultados de nuestra investigación hemos podido observar que el IMC tuvo reducción de 5,6\% en comparación con su valor inicial. El IMC, Glaner (2005) ha sido recomendado por la OMS como un indicador de la grasa corporal, porque se obtiene de manera rápida y prácticamente sin costo alguno. Esta también se obtiene de la medición de pliegues cutáneos y ha tenido gran aceptación entre los investigadores, ya que el porcentaje de grasa obtenida de la técnica antropométrica se asocia muy bien y no difiere significativamente del porcentaje derivados del pesaje hidrostático, que se toma como criterio de validación de otras técnicas.

En la tabla 1, están representadas otras variables en las que hubo disminución de pliegues cutáneos, sin embargo, no significativas. Después de ocho semanas de intervención hubo disminución del porcentaje de grasa. El pliegue cutáneo axilar bajó 12,91\% mientras que el pliegue cutáneo del tríceps braquial disminuyó $20,16 \%$ de la cantidad, medida inicialmente. El pliegue cutáneo pectoral tuvo reducción de $10,5 \mathrm{~mm}$, y con la menor tasa de reducción el pliegue cutáneo abdominal con $6.46 \%$ de grasa reducido. Los pliegues cutáneos de la región suprailíaca y muslo, obtuvieron la reducción más alta entre todos los otros pliegues. El muslo obtuvo una reducción de 12,25 milímetros sobre el valor tasado antes del programa de intervención, mientras que el pliegue suprailíaco obtuvo $16.2 \mathrm{~mm}$ de reducción, es decir, ambos los pliegues tienen tendencia a disminuir, mientras que para el muslo obtuvieron $\mathrm{p}=0,051 \mathrm{y}$ suprailíaco obtuvimos $p=0.058$. 
Tabla 1

Indicadores de la composición corporal de las personas antes y después del programa. Prueba t pareada.

\begin{tabular}{cccc}
\hline Pliegue cutáneo & Promedio $(\mathrm{mm})$ & Desviación estándar & $\mathrm{P}$ \\
\hline Axilar al inicio & 26,50 & 7,55 & 0.162 \\
Axilar al final & 23,08 & 7,22 & \\
Tríceps al inicio & 28,08 & 13.16 & 0.112 \\
Tríceps al final & 22,42 & 12,37 & 0,051 \\
Muslo al inicio & 30,17 & 12.10 & \\
Muslo al final & 17,92 & 4.821 & 0.058 \\
Suprailíaca al inicio & 37.33 & 16,59 & \\
Suprailíaca al final & 21,13 & 7,09 & 0.612 \\
Abdominal al inicio & 31.00 & 11,69 & \\
Abdominal al inicio & 29.00 & 5,96 & 0.102 \\
Pectoral al inicio & 27,17 & 14,55 & 6.86 \\
Pectoral al final & 16,67 & & \\
\hline
\end{tabular}

El pliegue subscapular se relaciona con resultados de estudios realizados por Mendes y Castro (2010) que investigaron la asociación entre actividad física y grasa corporal, utilizando la medida del pliegue cutáneo subscapular. Los resultados mostraron la intensa relación entre la práctica de actividad física y la grasa corporal subcutánea deposita en la región subscapular, revelando que cuanto más grande el esfuerzo físico, mayor será el número de células de grasa desplazados de la región subscapular.

Tabla 2

Dominios de indicadores de calidad de vida de las personas antes y después del programa. Emparejado t-test

\begin{tabular}{cccc}
\hline Dominios & Promedio $(\%)$ & Desviación estándar & P \\
\hline Físico al inicio & 72,61 & 17,44 & 0.10 \\
Física al final & 82.70 & 11,75 & \\
Psíquico al inicio & 57.94 & 7.25 & 0.01 \\
Psíquico al final & 72.88 & 7.87 & 0.02 \\
Social al inicio & 68.05 & 9,74 & \\
Social al final & 86.77 & 12,51 & 0.17 \\
Ambiental al inicio & 67.26 & 15.11 & 17,90 \\
Ambiental al final & 78.61 & &
\end{tabular}

En la tabla 2 se evidencia la CDV de la muestra encuestada. Se puede ver que hubo un aumento en todos los ámbitos estudiados, sin embargo, podemos destacar los dominios psíquicos y sociales donde obtuvieron diferencias significativas. La puntuación de dominio psíquico fue 58 y después de ocho semanas de intervención se observa un aumento a 73 , mientras el dominio social se extiende de 68 a 87 . Dominios físicos y ambientales también mejoraron, pero no con diferencias estadísticamente significativa.

En el análisis de CDV se observa un aumento en todas las áreas que mide, pero solo los dominios psíquicos y sociales obtuvieron resultado significativo; McArdle, Katch y Katch (2002) menciona que uno de los efectos del ejercicio físico es mejorar la CDV relacionada con la salud, mejorando el bienestar psicológico y permitiendo un buen funcionamiento físico en personas con una mala salud. Costa et al (2011) los compara a una buena nutrición con la $\mathrm{CDV}$, diciendo que la participación de programas que fomenten la realización de actividad física y consumo adecuado de la dieta nutricional puede reducir el riesgo de complicaciones de la enfermedad, además de contribuir a la mejora de la CDV de las enfermedades cardiovasculares. Esto refuerza la hipótesis de que el ejercicio físico, asociado con el estilo de vida, trae cambios en la composición corporal de las personas con obesidad o sobrepeso. 


\section{CONCLUSIONES}

Hemos observado la eficacia del programa de la disminución en los pliegues cutáneos, IMC y peso corporal. Sobre la relación entre la grasa y la mejor calidad de vida podemos percibir la mejora psíquica y social que los individuos han obtenido después del período de intervención.

\section{REFERENCIAS BIBLIOGRÁFICAS}

Associação Brasileira para o estudo da obesidade e da síndrome metabólica. Custos de Doenças Ligadas à Obesidade para o Sus. São Paulo; 2012.

Barbeau, P.; Litaker, M.S.; Woods, K.F.; Lemmon, C.R.; Humphries, M.C.; Owens, S. et al. (2002). Hemostatic and inflammatory markers in obese youths: effects of exercise and adiposity. J Pediatric, vol. 141, 415-420.

Barros, T.L. (2002). O Programa das 10 Semanas: uma proposta para trocar gorduras por músculos e saúde. Barueri: Manole.

Campos, M.O. E Rodrigues Neto, J.F. (2008). Qualidade de vida: um instrumento para a promoção de saúde. Revista Baiana de Saúde Pública, vol. 32, N. ${ }^{\circ}$, 232 - 240 maio/ago.

Cervato, A. M. et al. (1997). Dieta habitual e fatores de risco para doenças cardiovasculares. Revista de Saúde Pública, vol. 31, N. ${ }^{\circ}$ 3, 227-235.

Ciolac,E.G. E Guimaraes, G.V. (2004). Exercício físico e síndrome metabólica. Revista Brasileira de Medicina do Esporte, vol. 10, N. ${ }^{\circ}$ 4: 319-324, Jul/ Ago.

Costa, J. A. et al. (2011). Promoção da saúde e diabetes: discutindo a adesão e a motivação de indivíduos diabéticos participantes de programas de saúde. Ciência \& Saúde Coletiva, vol. 16, N. ${ }^{\circ} 3$,

Deliberato, Paulo C.P. (2002). Fisioterapia preventiva: fundamentos e aplicações. Barueri: Manole.

Ferreira, F. G. et al. (2010). Efeito do Nível de Condicionamento Físico e da Hidratação Oral sobre a Homeostase Hídrica em Exercício Aeróbico. Rev Bras Med Esporte, vol. 16, N. ${ }^{\circ}$ 3, 166-170, Mai/ Jun.

Ford, E.S.; Ajani, U.A.; Mokdad, A.H.(2005). The metabolic syndrome and concentrations of C-reactive protein among U.S. youth. Diabetes Care, N. ${ }^{\circ} 28$, vol. 4: $878-881$.

Foureaux, G; Pinto, K.M.C; Dâmaso, A. (2006). Efeito do consumo excessivo de oxigênio após exercício e da taxa metabólica de repouso no gasto energético. Revista Brasileira de Medicina do Esporte, vol. 12, N. ${ }^{\circ} 6,398$, Nov/Dez.
Glaner, M. (2005). Índice de massa corporal como indicativo da gordura corporal comparado às dobras cutâneas. Revista Brasileira de Medicina do Esporte, vol. 11, N. ${ }^{\circ} 4,243-246$.

Guerre-Millo M. (2004). Adipose tissue and adipokines: for better or worse. Diabetes Metab; vol. 30, N. $^{\circ} 1,13-19$.

Kisner, Carolyn E Colby, L.A. (2005). Exercícios terapêuticos: fundamentos e técnicas. 4. Ed. Barueri: Manole.

Lopes, Heno Ferreira. (2007). Hipertensão e inflamação: papel da obesidade. Revista Brasileira de Hipertensão, vol.14, N. ${ }^{\circ} 4,239-244$.

Marconi, M.A.; Lakatos, E.M. (2011). Metodologia do trabalho científico. 6. ed. São Paulo: Atlas.

Mcardle, W.D.; Katch, F.I. E Katch, V.L. (2008). Fisiologia do exercício: energia, nutrição e desempenho humano. 5. ed. Rio de Janeiro: Guanabara KooGan.

Mediano, M.F.F. et al. (2007). Efeito do Exercício Físico na Sensibilidade à Insulina em Mulheres Obesas Submetidas a Programa de Perda de Peso: Um Ensaio Clínico. Arquivos Brasileiros de Endocrinologia e Metabolismo, vol. 51, N. ${ }^{\circ} 6$.

Mendes, F.S.N.S. e Castro, C.L.B.(2010). Obesos Apresentam Menor Aderência a Programa de Exercício Supervisionado, Revista Brasileira de Cardiologia, vol. 23, N. ${ }^{\circ} 4,230-237$.

Milani RV, Lavie CJ, Mehra M. (2004) Reduction in C-reactive protein through cardiac rehabilitation and exercise training. Journal American Coll Cardiol. vol. 43, 1056-1061.

Oliveira, Ana Mayra A.; Cerqueira, Eneida M. M.; Souza, Josenira da Silva e Oliveira, A. C. (2003). Sobrepeso e Obesidade Infantil: Influência de Fatores Biológicos e Ambientais em Feira de Santana, BA. Arquivos Brasileiros de Endocrinologia e Metabolismo. vol. 47, N. ${ }^{\circ}$ 2, 144-150. Abril.

Perrella, M.; Marques P.; Rossi, L. (2005). Avaliação da perda hídrica durante treino intenso de rugby. Revista Brasileira de Medicina do Esporte, vol. 11, N. ${ }^{\circ}$ 4, 229-232, Jul/Ago. 
Rocca, S. V. S, Tirapegui, J; Melo, C. M.; Ribeiro, S. M. L. (2008). Efeito do exercício físico nos fatores de risco de doenças crônicas em mulheres obesas. Revista Brasileira de Ciências Farmacêuticas, vol. $44,{ }^{\circ}{ }^{\circ} 2,185-192$.

Rohlfs, I.C.P.M. et al. (2004). Aplicação de instrumentos de avaliação de estados de humor na detecção da síndrome do excesso de treinamento. Revista Brasileira de Medicina do Esporte, vol. 10, N. ${ }^{\circ}$ 2, 111-116, Mar/Abr.

Rossetti, M.B.; Britto, R.; Norton, R.C. (2009). Prevenção Primária de Doenças Cardiovasculares na Obesidade Infanto-juvenil: Efeito Anti-Inflamatório do Exercício Físico. Revista Brasileira de Medicina do Esporte, vol. 15, N. ${ }^{\circ} 6$ - Nov/Dez, 473.

Schneider, Ernst. A cura e a saúde pela natureza. Tatuí: Casa Publicadora Brasileira, 2005.
Wilmore, D.L. E Costill, J.H. (2009). Fisiologia do esporte e do exercício. 2. ed. Barueri: Manole, 2009.

World Health Organization. (1995). Physical status: the use and interpretation of anthropometry. Geneva; (WHO Technical Report Series, 854).

Zambon, L. et al. (2009). Efeitos de dois tipos de treinamento de natação sobre a adiposidade e o perfil lipídico de ratos obesos exógenos. Revista de Nutrição, vol. 22, N. ${ }^{\circ}$ 5, 707-715.

Zanette, C.A. et al. (2010). Síndrome metabólica e atrite idiopática juvenil. Revista Brasileira de Reumatologia, vol. 50, N. ${ }^{\circ}$ 2, 190-204.

Zucchi, P.; Nero \& Malik, A.M. (2000). Gastos em saúde: os fatores que agem na demanda e na oferta dos serviços de saúde, Saúde e Sociedade, vol. 9, N. ${ }^{1} / 2,127-150$. 